\title{
Joëlle Gardes Tamine, Poétique et rhétorique : la littérature et sa Belle Parole,
}

Paris, Honoré Champion, coll. «Bibliothèque de littérature générale et comparée », 2015, 288 p.

Kathryne Fontaine

Université de Toronto

À l'origine du dernier essai de Joëlle Gardes Tamine et, à vrai dire, de l'histoire littéraire française, une opposition: poétique et rhétorique. Puis, au fil des pages, ses dérivés : Belle Parole contre parole ordinaire, poésie contre discours et éloquence, fiction contre réalité, autant de concepts que les siècles et les critiques dressent les uns contre les autres, redéfinissent et rapprochent. La nature de la parole écrite est examinée dans nombre d'études, témoins du caractère problématique du sujet : existe-t-il vraiment du littéraire, puis du non littéraire? À une conception polarisée de 
la question, l'auteure de Poétique et rhétorique propose plutôt que « tout est affaire de degré et de continuum » (p. 270).

Le retour historique auquel elle procède en premier lieu fait davantage qu'éclairer le novice sur les relations entre poétique et rhétorique, lesquelles s'échangent au cours des siècles la position de prédominance dans le domaine du langage. Sa synthèse se concentre sur les périodes historiques qui présentent les plus marquantes distinctions, convergences et similitudes entre les deux disciplines. L'asymétrie des sections rapportant ces relations rejoint ainsi sa thèse initiale : davantage de points communs sont discernables entre poétique et rhétorique, et ce, même dans l'Antiquité, période que la tradition littéraire associe à une séparation marquée entre les deux. $\mathrm{Si}$, selon la conception de Michel Meyer, que G. Tamine reprend, la rhétorique est «la négociation de la distance entre des individus à propos d'une question donnée » (p. 32-333), la poétique participe aussi d'une sorte de marché entre l'écrivain et le lecteur et n'est en fin de compte qu'une autre « branche de l'emploi du langage » (p. 33).

\section{La voix propre au genre}

Poursuivant la réflexion d'A. Kibedi Varga, qui rapprocha le style lyrique du texte démonstratif sur le plan commun de la louange ou du blâme, c'est en détressant les unités constituantes des genres propres à la rhétorique ou à la poétique que G. Tamine arrive à en rattacher quelques-unes pour lier les deux pratiques, pour leur trouver des traits communs. Cette déconstruction la mène à déduire que, par exemple, ni la présence d'une question centrale, ni le médium choisi, ni l'espace, la situation externe, la 
volonté de plaire ou d'enseigner, ni même l'engagement de l'auteur ne constituent des critères infaillibles de classement des œuvres en genres. Les genres existent par les textes et les textes s'appuient sur les genres; les textes s'apparentent aux genres à divers degrés, et non pas par catégories exclusives. Cette démonstration efficace se poursuit avec l'exemple de la forme, constituant important de la poésie - que l'auteure nomme sa composition -, qui joue également un rôle clé dans le discours oratoire en tant que sa disposition. Le souci de la forme se retrouve donc dans les genres rhétoriques et littéraires, où il opère la même fonction : il nous aide à les repérer comme tels. C'est à la suite de ce raisonnement que l'auteure réussit à introduire la notion d'aptum, valeur fondamentale en rhétorique selon Marc Fumaroli et qui réfère à une adéquation entre le style, la matière et les thèmes d'un propos. Tout texte, nous dit-elle, a un rapport de soumission ou d'opposition à ce concept, autre preuve de «l'unité des usages du langage» (p.92). La difficulté demeure néanmoins de définir la littérature par rapport à la chose écrite qui n'en est pas, problème que résout temporairement G. Tamine en postulant qu' « un genre ne sera pas défini par un ensemble précis de traits, que tous les membres de la catégorie devraient partager, mais par une liste de propriétés que tous n'ont pas nécessairement» (p.94). En posant que les genres sont donc toujours modifiables et provisoires, et n'existent pas hors de la théorie, l'ouvrage se focalise sur un point crucial qui en fait toute son originalité et son importance : même si l'on place une œuvre à analyser dans un genre et que ce genre, par les caractéristiques des autres œuvres qui le constituent, parle pour cette œuvre, voire qu'il contribue à la définir, cette classification demeure secondaire. La méthode ici 
proposée est une analyse des "propriétés de tel ou tel phénomène [d'un texte], micro ou macrotextuel » (p. 104), à laquelle l'auteure procède par une typologie des genres, objets changeants qu'elle aborde par les transformations de leurs traits constituants.

Le texte passe donc à une revue des genres et de leur histoire, par le biais de retours en arrière et de projections vers l'avant, un récurrent «nous y reviendrons » ponctuant presque l'essai - mal nécessaire résultant du choix de la division de l'ouvrage? On se demande si les paramètres des genres et leur historique n'auraient pu être traités dans un seul bloc. On saisit néanmoins que les genres sont des catégories fluides qui ont fait l'objet de redéfinitions selon les points de vue des analystes et que leurs traits distinctifs demeurent, soit le caractère mimétique contre le caractère diégétique, le style, les conventions, la considération du public, etc. (p. 119) L'essai a ainsi l'ambition dialectique de vouloir remettre sous la loupe certains de ces traits et s'oppose à Genette («Frontières du récit», 1966) sur le sujet de la classification des termes mimésis et diégèsis chez Aristote et Platon que le critique, dans son essai de 1969, voyait comme un accord entre les deux philosophes antiques, sous une sémantique différente. G. Tamine démontre plutôt que leur définition des deux modes d'énonciation « diffère profondément » (p. 130) : «Chez Platon, la mimésis est un sousgenre de la diégèsis, alors que c'est l'inverse chez Aristote» (p. 130). Suivant Ricœur, l'auteure restitue à la notion de mimésis son sens de "construction de l'intrigue, du mythe» (p.131) et non de simple copie et soutient qu'au-delà de l'énoncé lui-même, c'est le mode d'énonciation et l'attitude de l'énonciateur qui importent. Le «qui parle» doit être posé, propos qui augure la partie IV de l'ouvrage, portant sur la fiction. Or cette 
interprétation force l'essayiste à faire un détour vers le lyrisme. En effet, la question de l'origine de l'énonciation (l'implication du locuteur dans le discours) permet d'explorer le problème du lyrisme, genre qui connut l'exclusion et l'inclusion de la Poétique - voire la glorification au rang de littérature pure - au fil du temps. Le lyrisme, affirme G. Tamine, n'est pas construit autour d'une intrigue. "Ainsi existe une séparation décisive du mimétique et du lyrique, même si l'expression de sentiments est aussi imitation de sentiments» (p.141). Le lyrisme présente également un énonciateur directement impliqué (présence du «je »). Bref, il exclut le narratif, propos habilement renforcé par les mots mêmes de Mallarmé : «Je veux [...] écrire comme elle frappa mon regard de poète, telle Anecdote, avant que la divulguent des reporters par la foule dressés à assigner à chaque chose son caractère commun» (p.150). S'appuyant sur Dominique Combe, l'étude montre que, par ce procédé d'exclusion de la forme narrative, on arrive à dépasser le vers tout en restant dans le poème, au moyen de la recherche de la spécificité (pureté) du langage poétique (p.151). Ainsi, le rapprochement essentiel que G. Tamine effectue entre mimétique narratif et lyrique - et si l'on revient à la question plus générale de son essai, entre rhétorique et poétique - , est celui du fait au virtuel. La poésie, au-delà de sa nature d'énonciation du personnel, tend vers une nécessité (p. 151). Elle clôt ce segment sur les genres en concluant que les différentes postures d'énonciation que prennent les écrivains jouent un rôle peu importe la nature d'un texte. Cette continuité est preuve ajoutée qu' «écriture » et «Belle Parole» s'inscrivent dans les mêmes paradigmes, menant éventuellement à une classification par genres, où ils sont distingués. 


\section{Qui dit vrai?}

L'une des principales distinctions que l'on fait entre poétique voire récit imaginaire - et le texte de non-fiction est de fait opérée sur ce plan du rapport à la réalité. Selon l'auteure, nous avons souvent tort d'appréhender ces deux notions dans un système dualiste : dès lors qu'est constatée la difficulté de définir le «réel», on comprend que la question ne concerne que l'existence des choses à travers le discours, à laquelle elle réfère par le concept d'ontologie (des textes). Dans cette optique, elle explique que les lettres procèdent d'une recherche du vraisemblable, d'une "modalité aléthique» (p.177), plutôt que d'une recherche de vérité. "Effet du nécessaire et de la rationalité, le vraisemblable est facteur de persuasion, ou plutôt, il est ce qui est plausible et répond aux attentes du récepteur » (p. 182) : c'est par cette définition du vraisemblable que le livre reconverge vers la rhétorique, observant que la considération de l'interlocuteur dans l'organisation du discours est un procédé commun aux deux disciplines. Évoquant bien sûr les critiques qui l'ont précédée sur ces questions, l'appareil argumentatif de G. Tamine n'en est pas moins original : devant Barthes affirmant que le réel est toujours sujet à la formulation qu'on en fait (p. 189-190), elle acquiesce et complète que la séparation des discours factuels ou fictifs est subtile : si le fait y joue un rôle fondateur, elle classe le texte dans le factuel; s'il est le résultat du récit, il en va de fiction. À la suite de Ricœur (Temps et récit, 1983-1985) posant que l'histoire a une fonction de «représentance » du réel, et la fiction un rôle de «signifiance» (190), elle poursuit que le langage, situé entre nous et le monde, limite la manière de décrire ce monde. Pour les écrivains, cette limite est en fait une libération, car c'est dans son caractère 
inéluctablement divergent de la réalité que le langage tire toute sa force autonome. Reprenant le terme de Molinié (2005), elle entreprend d'expliquer que les dispositifs de la langue traitant du contact entre la réalité et la conception que l'on s'en fait mènent à l'apparition $\mathrm{du}$ mondain, terme technique soigneusement sélectionné pour appuyer le reste de son argumentation. Citant Saint-John Perse (1972) - «toute poétique est une ontologie »-, elle fait le lien entre ce mondain où se fabrique le sens d'un objet narratif, et la poésie lyrique qui permet au lecteur de « connaître et de nommer des sentiments informulés » (p. 220). Puis, continuant d'interroger les modes d'existence des objets du texte, elle fait appel aux récentes théories d'Étienne Souriau (Les Différents Modes d'existence, 2012) pour faire un bref détour du côté de la philosophie. Même si l'on doute que ce segment soit aisément inséré dans le fil de l'argumentation, la seule lecture de ce paragraphe assez technique pouvant difficilement permettre de comprendre Souriau (le vocabulaire, déjà, est fort ésotérique - phénomènes en patuité, réiques, sollicitudinaires... (p. 224) -, on entend que G. Tamine ait voulu montrer le discutable bien-fondé de l'opposition entre réalité et fiction et réaffirmer que l'œuvre d'art a sa réalité propre, qui justifie ses usages et son intérêt. Bref, il n'y aurait tout simplement pas de légitimité à opposer les mondes de la fiction et du réel de manière tranchée. Si la fiction opère également dans le domaine du possible, le monde empiriquement supposé réel perd son titre de référent factuel auquel comparer les autres. Tout est invention, et c'est dans la manière de faire passer l'inexistence - G. Tamine dit l'absence à la présence que le langage se détermine. 
La langue fait donc davantage que transmettre un message. Celui-ci prend son sens et sa forme à mesure que le langage se construit, «dans la relation ethos-logos » et dans sa réception, «entre le logos et le pathos» (p. 246). Dans cette optique, la rhétorique s'avère inhérente à l'utilisation du langage. En effet, l'auteure signale, conformément au principe d'un tout plus grand que la somme de ses parties, que les signes contiennent en eux-mêmes une référence générale avant d'être mis dans un contexte déterminé. Ce poids sémantique confère au langage sa nature argumentative. Tentant enfin quelques définitions de son cru pour saisir l'essence de la littérarité d'un texte, G. Tamine rappelle la théorie de la problématologie de Meyer, qui cherche à déterminer la question sous-jacente d'un texte lorsqu'on en fait l'interprétation. Savoir qu'il y a quelque chose à déceler sans savoir ce que cela est exactement, dans un discours comme dans un poème, indique la nature littéraire de l'objet. Toute la pertinence de l'ouvrage se résume dans la constatation qu'il y a toujours présence de discours entre une réalité et la conception que l'on s'en fait. L'esprit humain navigue à travers des représentations de son entourage, et non de «vérités désincarnées » (p. 270). Ces représentations agissent selon la subjectivité du lecteur et leur appréhension est continuellement modifiée par le renouvellement des récits et des questions. Montrer que poétique ou rhétorique cesse d'être le seul paradigme par lequel aborder un texte; voilà la gageure de l'essai de G. Tamine. En fait, si des mentions répétitives du besoin de revenir à une notion ou à une autre parsèment l'ouvrage, ils ont pour effet positif de mener naturellement la lecture vers la conclusion qui, une fois le corps du texte assimilé, apparaît de soi. 